Advance Access publication 2017 April 11

GJI Geomagnetism, rock magnetism and palaeomagnetism

\title{
The dispersion of magnetic-Coriolis waves in planetary cores
}

\author{
O.P. Bardsley and P.A. Davidson \\ Department of Engineering, University of Cambridge, Trumpington Street, Cambridge, CB2 1PZ, United Kingdom.E-mail: pad3@eng.cam.ac.uk
}

Accepted 2017 April 10. Received 2017 March 31; in original form 2017 January 19

\begin{abstract}
S U M M A R Y
We consider the dispersion of waves in a rapidly rotating, Boussinesq fluid which is threaded by a magnetic field and stirred by slowly gravitating buoyant blobs. Motivated by dynamics in the core of the Earth, we focus on the evolution of inertial-Alfvén wave packets radiated from the buoyant anomalies. These waves resemble conventional low-frequency inertial waves, in the sense that energy disperses on the fast timescale of the background rotation rate, though they also exhibit slower Alfvén-like propagation along magnetic field lines. When the magnetic field is uniform, inertial-Alfvén waves automatically focus energy radiation onto the rotation axis, a property they share with conventional low-frequency inertial waves in the hydrodynamic case, and which ensures that they dominate the dispersion pattern. However, the situation changes significantly when the magnetic field, $\mathbf{B}$, is non-uniform. In particular, we show any non-uniformity of $\mathbf{B}$ causes inertial-Alfvén waves to evolve into a more general form of magnetic-Coriolis (MC) wave, and that these waves refract, dispersing somewhat offaxis. Moreover, if inertial-Alfvén waves are launched near the equator they can be confined to low latitudes by a critical layer at which the axial group velocity drops to zero. Given that the magnetic field in a planetary core is inevitably non-uniform, we conclude that quasi-geostrophy is most likely achieved through a combination of weakly modified inertial waves and a form of slightly off-axis MC wave in which the inertial and Alfvén frequencies are comparable.
\end{abstract}

Key words: Core; Dynamo: theories and simulations; Geomagnetic induction; Planetary interiors; Rapid time variations.

\section{INTRODUCTION: INERTIAL AND INERTIAL-ALFVÉN WAVES IN A RAPIDLY ROTATING FLUID}

Incompressible internal waves play a potentially important role in magnetic field generation within planetary cores (Moffatt 1970; Olson 1981). In particular, they are effective at generating and then transporting the much needed helicity, $h=\mathbf{u} \cdot \nabla \times \mathbf{u}$, within a planetary core, and in such a way that it is skew-symmetric about the equator, as required by classical dynamo theory (Davidson 2014; Davidson \& Ranjan 2015). These waves can be maintained by both the magnetic field (Alfvén waves) and the background rotation (inertial waves), though it is likely in a planetary core that the Coriolis and Lorentz forces act in unison to produce some kind of hybrid magnetic-Coriolis (MC) wave. Perhaps the most frequently investigated regime is that in which the Alfvén frequency is significantly smaller than the inertial wave frequency, as is traditionally assumed for the Earth's core, in which rapid rotation dominates the comparatively weak magnetic field. The resulting waves are then usually classified in one of two ways: weakly modified inertial waves and the so-called magnetostrophic waves (Moffatt 1978; Finlay et al. 2010). Recently, however, Bardsley \& Davidson (2016) noted that, for Earth-like parameters, there exists a third class of waves for which the inertial wave frequency is in fact much smaller than the Alfvén frequency. When the magnetic field is uniform this new class of waves is particularly efficient at dispersing energy along the rotation axis and so helps promote quasi-geostrophy in a stirred, rotating fluid. These waves represent a form of hybrid motion, which retains certain features of both inertial and Alfvén waves, and so Bardsley \& Davidson (2016, hereafter BD16) called them inertialAlfvén waves. In order to place this study in perspective, introduce some notation, and establish the relevant governing equations, it is useful to briefly summarize the properties of these various classes of waves.

Consider a rapidly rotating, Boussinesq fluid stirred by slowly gravitating buoyant anomalies of scale $\ell$ and threaded by a uniform magnetic field $\mathbf{B}_{0}$. We adopt a system of co-ordinates which rotates with the fluid and the field is taken to be steady in the rotating frame. To focus thoughts we take the background rotation $\boldsymbol{\Omega}=\boldsymbol{\Omega} \mathbf{e}_{z}$, the magnetic field, and the gravitational acceleration $\mathbf{g}$ to be mutually orthogonal. (In the case of the Earth, $\mathbf{B}_{0}$ might be the local east-west field in the equatorial regions.) If we consider small perturbations in the rotating frame, and ignore buoyancy forces and background motion, the linearized equations of motion for an ideal fluid are

$$
\begin{aligned}
\frac{\partial \mathbf{u}}{\partial t} & =\frac{1}{\rho \mu}\left(\mathbf{B}_{0} \cdot \nabla\right) \mathbf{b}+(2 \boldsymbol{\Omega} \cdot \nabla) \mathbf{c}, \\
\frac{\partial \mathbf{b}}{\partial t} & =\left(\mathbf{B}_{0} \cdot \nabla\right) \mathbf{u},
\end{aligned}
$$


where $\rho$ and $\mu$ are the density and permeability of free space, $\mathbf{u}$ and $\mathbf{b}$ the velocity and perturbed magnetic fields, and $\mathbf{c}$ the solenoidal vector potential for $\mathbf{u}$. These combine to give the wave-like equation

$\left[\frac{\partial^{2}}{\partial t^{2}}-\frac{1}{\rho \mu}\left(\mathbf{B}_{0} \cdot \nabla\right)^{2}\right]^{2} \nabla^{2} \mathbf{u}+(2 \boldsymbol{\Omega} \cdot \nabla)^{2} \frac{\partial^{2} \mathbf{u}}{\partial t^{2}}=0$.

If we search for solutions of the form $[\mathbf{u}, \mathbf{b}]=$ $[\hat{\mathbf{u}}, \hat{\mathbf{b}}] \exp \{i(\mathbf{k} \cdot \mathbf{x}-\varpi t)\}$, we find several classes of waves. In the absence of a magnetic field we obtain inertial waves, which have angular frequency $\varpi= \pm 2 \boldsymbol{\Omega} \cdot \boldsymbol{k} / k$, group velocity $\mathbf{c}_{g}= \pm 2 k^{-3}(\mathbf{k} \times(\boldsymbol{\Omega} \times \mathbf{k}))$, and maximal helicity $h=\mathbf{u} \cdot \nabla \times \mathbf{u}$. On the other hand, with no background rotation we obtain non-dispersive Alfvén waves whose frequency and group velocity are $\varpi=( \pm) \mathbf{B}_{0} \cdot \mathbf{k} / \sqrt{\rho \mu}$ and $\mathbf{c}_{g}=( \pm) \mathbf{B}_{0} / \sqrt{\rho \mu}$ respectively. More generally, for finite $\mathbf{B}_{0}$ and $\boldsymbol{\Omega}$, we obtain the dispersion relationship

$\varpi^{2} \mp \varpi_{\Omega} \varpi-\varpi_{B}^{2}=0$,

where $\varpi_{B}=\mathbf{B}_{0} \cdot \mathbf{k} / \sqrt{\rho \mu}$ and $\varpi_{\Omega}=2 \boldsymbol{\Omega} \cdot \boldsymbol{k} / k$. The corresponding amplitudes are readily shown to satisfy

$\hat{\mathbf{u}}=\mp \frac{\hat{\boldsymbol{\omega}}}{k}, \quad \hat{\mathbf{a}}=\mp \frac{\hat{\mathbf{b}}}{k}, \quad \hat{\mathbf{u}}=-\frac{\varpi}{\varpi_{B}} \frac{\hat{\mathbf{b}}}{\sqrt{\rho \mu}}$,

$\boldsymbol{\omega}$ being the vorticity and $\mathbf{a}$ the (solenoidal) vector potential for $\mathbf{b}$ (see, e.g., Moffatt 1978). Evidently, $\mathbf{u}$ and $\omega$ are in phase and parallel, so that all plane-wave solutions have maximal kinetic helicity $h=\mathbf{u} \cdot \omega$, with the upper (lower) sign in (4) corresponding to negative (positive) helicity. Moreover, the fact that $k \hat{\mathbf{u}}=\mp \hat{\boldsymbol{\omega}}$, irrespective of the presence of a magnetic field, tells us that all monochromatic solutions have the same spatial structure for $\mathbf{u}$ (Moffatt 1978), while $k \hat{\mathbf{a}}=\mp \hat{\mathbf{b}}$ tells us that the magnetic helicity $h_{m}=\mathbf{a} \cdot \mathbf{b}$ is also maximal.

The group velocity corresponding to (4) can be expressed as

$\left(1+\frac{\varpi_{B}^{2}}{\varpi^{2}}\right) \mathbf{c}_{g}=\frac{2 \varpi_{B}}{\varpi} \frac{\mathbf{B}_{0}}{\sqrt{\rho \mu}} \pm \mathbf{c}_{g \Omega}$,

where $\mathbf{c}_{g \Omega}=2\left(\boldsymbol{\Omega}-\left(\mathbf{e}_{k} \cdot \boldsymbol{\Omega}\right) \mathbf{e}_{k}\right) / k$ and $\mathbf{e}_{k}=\mathbf{k} / k$. Note that all wave packets propagating in the direction of $\boldsymbol{\Omega}$ carry negative helicity, while those propagating antiparallel to $\boldsymbol{\Omega}$ carry positive helicity. Note also that all wavevectors lying in the horizontal plane disperse energy along the rotation axis through the term $\mathbf{c}_{g \Omega}$.

Now the small scales in the geodynamo are expected to operate in a regime in which $\Omega \ell$ is greater than $\sim 30|\mathbf{B}| / \sqrt{\rho \mu}$, where we take $\ell \sim 10 \mathrm{~km}$ and assume a field of around 30 Gauss (consistent with Gillet et al. 2010). So, if we set aside the special case of $\boldsymbol{\Omega} \cdot \boldsymbol{k} \approx 0$, then $\left|\varpi_{\Omega}\right| \gg\left|\varpi_{B}\right|$ and the dispersion relationship (4) yields two distinct pairs of roots, $\varpi \approx \pm \varpi_{\Omega}$ and $\varpi \approx \mp \varpi_{B}^{2} / \varpi_{\Omega}$. The latter represents a magnetostrophic wave, which is characterized by a particularly low frequency and, by virtue of (6), a slow group velocity. However, this is not the only possibility. We know from studies of rapidly rotating, non-magnetic turbulence that the special case of $\boldsymbol{\Omega} \cdot \boldsymbol{k} \approx 0$ plays a crucial role in the formation of columnar vortices. To understand this, consider a localized disturbance of arbitrary shape in a non-conducting fluid which excites inertial waves with a spectrum of wavevectors $\mathbf{k}$. The direction of propagation of the resulting wave energy will vary, fixed by the orientation of $\mathbf{k}$ through $\mathbf{c}_{g}= \pm \mathbf{c}_{g \Omega}$. However, the fact that $\mathbf{c}_{g}$ is perpendicular to $\mathbf{k}$ means that all waves which satisfy $\boldsymbol{\Omega} \cdot \boldsymbol{k} \approx 0$ must propagate in the direction of $\boldsymbol{\Omega}$, and so all the energy contained in a thin horizontal disc in k-space will be focussed onto, and then propagate along, the rotation axis. This concentrates the radiated energy from a localized source onto the rotation axis, so that the energy density in axially propagating wave packets is higher than in off-axis ones (Davidson et al. 2006).

BD16 noted that essentially the same phenomenon will occur in the presence of a magnetic field, even in the Earth-like regime where one might normally expect to find $\left|\varpi_{\Omega}\right| \gg\left|\varpi_{B}\right|$. In particular, they considered those wave packets which satisfy $\boldsymbol{\Omega} \cdot \boldsymbol{k} \approx 0$, so that the frequencies are ranked according to $\left|\varpi_{\Omega}\right| \ll\left|\varpi_{B}\right|$, despite the fact that $\Omega \ell \gtrsim 30|\mathbf{B}| / \sqrt{\rho \mu}$. The key idea is that, like low-frequency inertial waves in the strictly hydrodynamic case, the constraint that $\mathbf{k}$ is perpendicular to $\mathbf{c}_{g \Omega}$, combined with $\boldsymbol{\Omega} \cdot \boldsymbol{k} \approx 0$, ensures that wave energy dispersing from a localized source is focussed preferentially onto the rotation axis.

The various properties of these waves are readily obtained from (4) and (5). For $\boldsymbol{\Omega} \cdot \boldsymbol{k} \approx 0$, and to leading order in $\left|\varpi_{\Omega}\right| /\left|\varpi_{B}\right|$, these yield

$\varpi=( \pm) \varpi_{B} \pm \frac{\varpi_{\Omega}}{2} \approx( \pm) \varpi_{B}, \quad \hat{\mathbf{u}}=(\mp) \frac{\hat{\mathbf{b}}}{\sqrt{\rho \mu}}$,

where the sign in $( \pm)$ may be chosen independently from that in \pm . The corresponding group velocity is

$\mathbf{c}_{g}=( \pm) \frac{\mathbf{B}_{0}}{\sqrt{\rho \mu}} \pm \frac{\boldsymbol{\Omega}}{k}$.

Evidently these are equipartition waves which have the frequency of an Alfvén wave, yet disperse energy along the rotation axis at half the group speed of low-frequency (i.e. $\boldsymbol{\Omega} \cdot \boldsymbol{k} \approx 0$ ) inertial waves. For Earth-like parameters the axial group velocity of these waves is fast, traversing the core on a timescale of months, which might be compared with the speed of Alfvén waves, which traverse the core in years to decades. Physically, the waves may be considered a modified version of the low-frequency $(\boldsymbol{\Omega} \cdot \boldsymbol{k} \approx 0)$ inertial waves which are responsible for the columnar axial wave packets in the strictly hydrodynamic case. The key difference is that, when $\mathbf{B}$ is finite, an axially propagating wave packet needs to bend magnetic field lines and this drops the axial group velocity by a factor of two, while the helical flow within the wave packet spirals up the field lines yielding a finite magnetic and cross helicity. Finally, the presence of the Alfvén frequency in (7) and group velocity in (8) tells us that the bending of the field lines also initiates slower, horizontally propagating Alfvén waves. (The initiation of slow, horizontal Alfvén waves by fast inertial waves is also observed in Jault (2008).)

This general behaviour is readily confirmed via a simple example: BD16 consider a localized buoyant blob sitting in an ideal, rotating fluid which is permeated by a uniform magnetic field. Through an analytical Fourier-transform solution, they show that the buoyant anomaly spontaneously radiates axially elongated wave packets which propagate along the rotation axis and evolve into quasi-geostrophic vortices. This is illustrated in Fig. 1(a), which shows the dispersion of wave energy from a small Gaussian-shaped buoyant anomaly of scale $\ell$, which is introduced at the origin at $t=0$. (In this case $\boldsymbol{\Omega}$ is vertical, gravity acts into the page, $\mathbf{B}_{0}$ points from left to right, and $\left(B_{0} / \sqrt{\rho \mu}\right) / \Omega \ell=0.05$.) While some off-axis radiation is evident, in the form of oblique inertial waves, the dispersion pattern is dominated by axially propagating inertialAlfvén waves as expected. At yet larger times the horizontal propagation is more pronounced, but the dispersion pattern continues to be dominated by the columnar wave packets above and below the buoyant anomaly. The corresponding wave pattern in the absence of a magnetic field is shown in Fig. 1(b), which is also dominated by dispersion along the rotation axis, in this case composed of low 
(a)

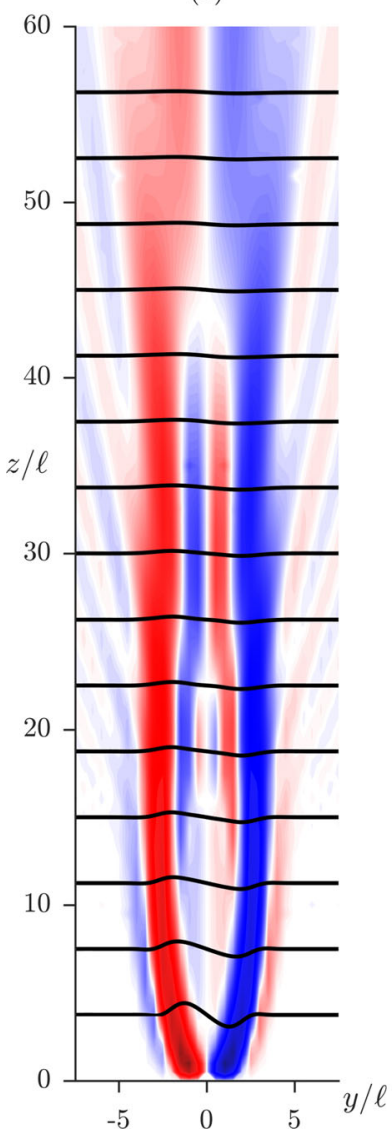

(b)

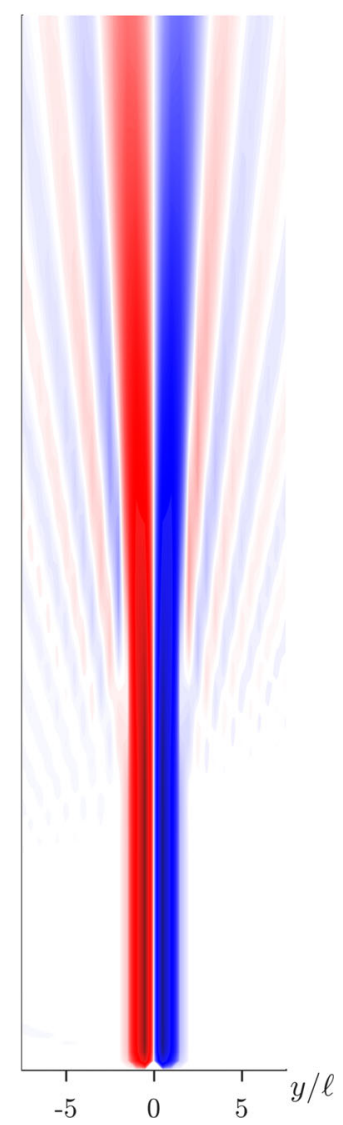

Figure 1. The dispersion pattern from a small, localized buoyant anomaly of scale $\ell$ located at the origin. $\boldsymbol{\Omega}$ is vertical, B points from left to right, and $\mathrm{g}$ acts into the page. The axial velocity is shown at $\Omega t=50$, with red (blue) signifying $u_{z}>0\left(u_{z}<0\right)$, and magnetic field lines are overlaid in black. (a) $\left(B_{0} / \sqrt{\rho \mu}\right) / \Omega \ell=0.05$, (b) $B_{0}=0$. From BD16.

frequency $(\boldsymbol{\Omega} \cdot \boldsymbol{k} \approx 0)$ inertial waves. (The wave dispersion in the non-magnetic case is discussed in Davidson (2014).)

In summary, then, hybrid inertial-Alfvén waves are particularly efficient at generating axially elongated vortices (wave packets) from localized buoyant anomalies and so can help maintain approximate geostrophy in a continually stirred, rotating fluid. However, a crucial limitation of the analysis in BD16 is that the magnetic field is assumed to be spatially uniform, whereas $\mathbf{B}$ is clearly non-uniform in the core of a planet. We now show that any non-uniformity of B disrupts the ability of inertial-Alfvén waves to focus energy onto the rotation axis. Indeed, in certain circumstances, these waves are confined to low latitudes and cannot carry any energy into the polar regions. In any case, inertial-Alfvén waves cease to play any special role in establishing quasi-geostrophy as they evolve into more general MC waves.

\section{SPONTANEOUS RADIATION FROM A LOCALIZED SOURCE WHEN THE MAGNETIC FIELD VARIES WITH LATITUDE}

\subsection{Ray tracing in an inhomogeneous magnetic field: a model problem}

We consider a model problem in which waves radiate from a single localized source, say a buoyant blob of scale $\ell$, introduced into

the fluid at time $t=0$. We restrict ourselves to the regime $\Omega \ell \gg$ $|\mathbf{B}| / \sqrt{\rho \mu}$, which is thought typical of the Earth's core, and consider the case where $\boldsymbol{\Omega}$ and $\mathbf{B}_{0}$ are mutually orthogonal, with $\boldsymbol{\Omega}=\boldsymbol{\Omega} \mathbf{e}_{z}$ and $\mathbf{B}_{0}=B_{0} \mathbf{e}_{y}$. Moreover we take $\mathbf{B}_{0}$, which we may think of as the east-west field in the core of the Earth, to be anti-symmetric about the equator $(z=0)$ and to vary slowly with $z$. (By slowly varying we mean that $\mathbf{B}_{0}$ changes negligibly on the scale of $k^{-1}$, i.e. $k L \gg 1$.) So we write $B_{0}(z)=B^{*} f(z / L)$, where $f$ is an odd function in $z / L$ and $B^{*}$ and $L$ are constants which are indicative of the peak east-west field strength and core radius in the Earth. If we restrict ourselves to moderate-to-low latitudes, say $|z|<L / 2$, then we might take $f$ as linear, say $B_{0}(z)=B^{*} z /(L / 2)$. However, the azimuthally averaged east-west field falls back down to zero at the mantle, so for a broader range of latitudes, say $|z|<L$, the form $B_{0}(z)=B^{*} \sin (\pi z / L)$ might be more appropriate. Finally, since $z=0$ is an atypical location characterized by zero magnetic field, we let the source sit at an arbitrary value of $z$, say $z=z_{0}$, and not be restricted to the equator, though we have in mind locations not too far from it.

Note that the spatial variation of $\mathbf{B}_{0}$ implies the existence of a background current density, $\mathbf{J}_{0}=\mu^{-1} \nabla \times \mathbf{B}_{0}$. This introduces additional terms into the perturbation equations (1) and (2). However, it is ready confirmed that, in the limit of $k L \gg 1$, the resulting current is weak and these extra terms are second order in $(k L)^{-1}$ and so may be neglected in the analysis that follows.

Although the problem is now inhomogeneous in $z$, the slow variation in $\mathbf{B}_{0}$ means that we may still look for local plane-wave solutions of the form $[\mathbf{u}, \mathbf{b}]=[\hat{\mathbf{u}}, \hat{\mathbf{b}}] \exp \{i(\mathbf{k} \cdot \mathbf{x}-\varpi t)\}$. We can then use conventional ray-tracing methods (Lighthill 1978) to track the evolution of the various wave packets emanating from the buoyant anomaly as they propagate away from the source. The local dispersion relationship is then (4), which is conveniently rewritten as

$\frac{\varpi_{\Omega}}{\varpi}= \pm\left(1-\frac{\varpi_{B}^{2}}{\varpi^{2}}\right)$,

where $\varpi_{B}=\mathbf{B}_{0} \cdot \mathbf{k}_{\perp} / \sqrt{\rho \mu}, \varpi_{\Omega}=2 \Omega k_{\|} / k$, and the subscripts $\perp$ and $\|$ indicate the orientation of $\mathbf{k}$ relative to $\boldsymbol{\Omega}$. Note that the upper sign in (9) corresponds to northward propagating wave packets and the lower sign to southward propagation.

According to standard ray-tracing theory the frequency of a wave packet is always conserved. Moreover, because our model problem is homogeneous in planes normal to $\boldsymbol{\Omega}$, the horizontal wavevector $\mathbf{k}_{\perp}$ is also preserved by a wave packet (Lighthill 1978). It follows that we may write (9) in the more useful form

$$
\begin{aligned}
& \frac{\varpi_{\Omega}(z)}{\varpi_{0}}=\frac{2 \Omega k_{\|} / k}{\varpi_{0}}= \pm\left(1-\frac{\varpi_{B}^{2}(z)}{\varpi_{0}^{2}}\right), \\
& \varpi_{B}(z)=\frac{\mathbf{B}_{0}(z) \cdot \mathbf{k}_{\perp 0}}{\sqrt{\rho \mu}},
\end{aligned}
$$

where $\varpi_{0}$ and $\mathbf{k}_{\perp 0}$ are the initial launch values of $\varpi$ and $\mathbf{k}_{\perp}$. Evidently, in our model problem, we may consider $\varpi_{B}$ to be a specified function of $z$ and our dispersion relationship then tells us how $\varpi_{\Omega}(z)$, and hence $k_{\|}$, varies as the wave packet propagates through the magnetic field.

Relationship (10) is conveniently plotted as shown in Fig. 2, where for clarity we show only the upper branch corresponding to northward propagating waves. Any wave packet launched from the buoyant blob then follows the downward sloping dispersion curve in Fig. 2, as it pushes northward into regions of stronger $\mathbf{B}_{0}$. The left-hand part of the dispersion curve corresponds to low values of $\mathbf{B}_{0}$ and to weakly modified inertial waves, $\varpi_{0} \approx \varpi_{\Omega}$, while the right-hand part leads to ever stronger magnetic fields and eventually 


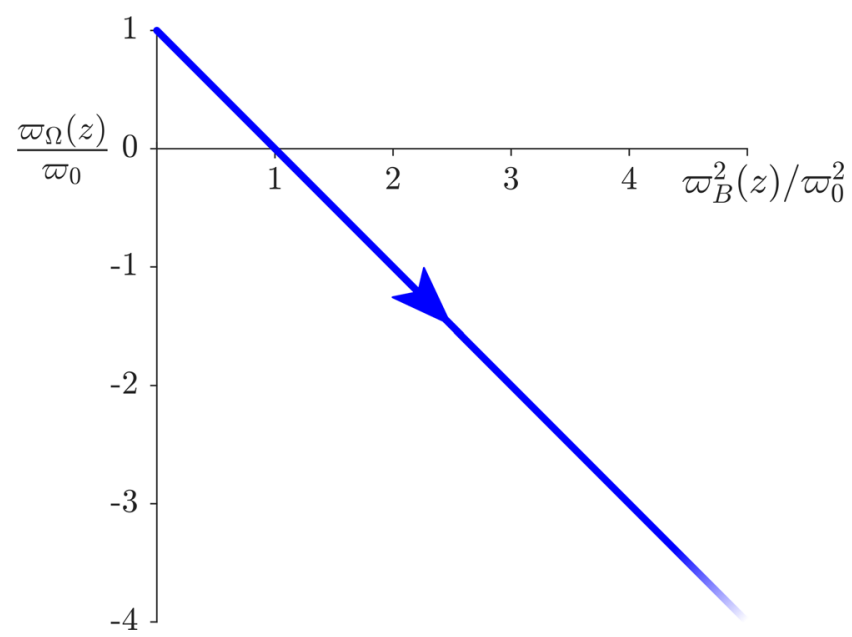

Figure 2. The variation of $\varpi_{\Omega}(z) / \varpi_{0}$ with $\varpi_{B}^{2}(z) / \varpi_{0}^{2}$ according to the dispersion relationship (10). For simplicity, only the upward propagating branch is shown.

to magnetostrophic waves, $\varpi_{0} \approx-\varpi_{B}^{2} / \varpi_{\Omega}$. The hybrid inertialAlfvén waves of BD16 evidently correspond to the crossover point where $k_{\|}=0$ and $\varpi_{0}=\varpi_{B}$.

We now show that this slow variation in the background field disrupts the ability of inertial-Alfvén waves to focus wave energy onto the rotation axis, and indeed in some circumstances inertialAlfvén waves are confined to the equatorial regions.

\subsection{The refraction of wave packets and the wave ceiling}

Let us now consider the dispersion pattern associated with a single source. It is important to note that, while $\left|\mathbf{k}_{\perp 0}\right|$ in (10) presumably scales with the inverse size of the buoyant anomaly, and so can be estimated a priori, the launch frequency $\varpi_{0}$ cannot be similarly determined. Rather, there will be a range of launch frequencies dictated by the initial distribution of wave energy in $\mathbf{k}$-space, with each frequency corresponding to the local dispersion of wave energy in a particular direction. In short, the launch conditions will resemble those shown in Fig. 1(a). Hence a single buoyant anomaly will radiate energy corresponding to a range of orientations of $\mathbf{k}$, or equivalently a range of values of $\varpi_{0}$, and thus to a range of values of $\varpi_{B}^{2}(z) / \varpi_{0}^{2}$ in Fig. 2. However, the dispersion of energy adjacent to the buoyant anomaly, where the field looks locally uniform, will be dominated by axially propagating hybrid inertial-Alfvén waves, as shown in Fig. 1(a). So we shall focus particularly on launch conditions corresponding to the crossover point $k_{\|}=0$ and $\varpi_{0}=\varpi_{B}$ in Fig. 2. (It is evident from Fig. 1(a) that off-axis weakly modified inertial waves also play a significant role in transporting wave energy along the rotation axis, but we shall set this aside for the moment.) Our task now is to determine what happens to axially propagating wave packets as they pass upward into regions of stronger magnetic field.

For such wave packets we have $\varpi_{0}=\varpi_{B}\left(z_{0}\right)$ and so (10) becomes

$$
\frac{\varpi_{\Omega}}{\varpi_{0}}=\frac{2 \Omega k_{\|} / k}{\varpi_{B}\left(z_{0}\right)}= \pm\left(1-\frac{\varpi_{B}^{2}(z)}{\varpi_{B}^{2}\left(z_{0}\right)}\right)= \pm\left(1-\frac{B_{0}^{2}(z)}{B_{0}^{2}\left(z_{0}\right)}\right) .
$$

It is immediately apparent that, while $k_{\|}=0$ at $z=z_{0}$, it is non-zero for $z \neq z_{0}$. We conclude that hybrid inertial-Alfvén waves, which are characterized by $\boldsymbol{\Omega} \cdot \boldsymbol{k} \approx 0$, will not remain inertial-Alfvén waves for long, but rather evolve into off-axis wave packets with a finite value of $k_{\|}$. (Recall that $\mathbf{c}_{g \Omega}$ is perpendicular to $\mathbf{k}$.) Thus the ability of inertial-Alfvén waves to focus energy onto the rotation axis, which rests on the property $k_{\|} \approx 0$, cannot be maintained when $\mathbf{B}_{0}$ is non-uniform.

The ultimate fate of these wave packets depends on where the waves are launched, that is, the location of the buoyant anomaly. Away from the equator we have the estimate

$$
\frac{\Omega}{\varpi_{B}\left(z_{0}\right)} \sim \frac{\Omega \ell}{B^{*} / \sqrt{\rho \mu}}
$$

which is usually taken to be large in the core of the Earth (greater than 30). Provided that the magnitude of $B_{0}(z)$ is never very different from that at launch, $B_{0}\left(z_{0}\right)$ (an obvious exception being wave packets launched near the equator), a large value of $\Omega / \varpi_{B}\left(z_{0}\right)$ means that $\left|k_{\|}\right| / k$ in (11) will remain relatively modest. We conclude that many (but certainly not all) wave packets continue to have a group velocity which is approximately aligned with the rotation axis.

This is illustrated by the rays shown in Fig. 3(a) for the particular case in which $B_{0}(z)=2 B^{*} z / L, \ell \sim \pi /\left|\mathbf{k}_{\perp}\right|=10 \mathrm{~km}, L=2000 \mathrm{~km}$, the rotation rate is that of the Earth, the normalized field strength is $\left(B^{*} / \sqrt{\rho \mu}\right) / \Omega \ell=0.033\left(B^{*}=30 \mathrm{G}\right)$, and the launch position is $z_{0}=500 \mathrm{~km}$. These trajectories are plotted by integrating the relationships

$\frac{\mathrm{d} z}{\mathrm{~d} t}=c_{g z}(z), \frac{\mathrm{d} x}{\mathrm{~d} z}=\frac{c_{g x}(z)}{c_{g z}(z)}, \frac{\mathrm{d} y}{\mathrm{~d} z}=\frac{c_{g y}(z)}{c_{g z}(z)}$,

for the equations of the rays. The various components of the group velocity are given by substituting (10) into (6). The circles on the rays indicate that $0.1 \mathrm{yr}$ have passed since the waves were launched.

Note that, although $\left|k_{\|}\right| / k$ remains small in such a case, it is unreasonable to suppose that the condition $\left|\varpi_{\Omega}\right| \ll\left|\varpi_{B}\right|$ is maintained as the wave packets push north and south. Rather, we would expect the right-hand side of (11) to be of order unity, so that in general $\left|\varpi_{\Omega}\right| \sim\left|\varpi_{B}\right|$. It follows that, although $\mathbf{c}_{g \Omega}$ often remains approximately aligned with the rotation axis, these waves cannot continue to be classified as hybrid inertial-Alfvén waves, governed by (7) and (8).

There are, however, certain wave packets for which $\left|k_{\|}\right| / k$ does not remain small and so $\mathbf{c}_{g \Omega}$ becomes significantly misaligned with $\boldsymbol{\Omega}$. This is particularly relevant for waves launched close to the equator, and in such cases there exists the possibility that the waves remain trapped in the equatorial regions, as we now discuss. Eq. (11) in the form

$$
\left|\frac{B_{0}^{2}(z)}{B_{0}^{2}\left(z_{0}\right)}-1\right|=\left|\frac{2 \Omega k_{\|} / k}{\varpi_{B}\left(z_{0}\right)}\right|<\frac{2 \Omega}{\varpi_{B}\left(z_{0}\right)}
$$

tells us that, when $B_{0}\left(z_{0}\right) / B^{*}$ is particularly small (i.e. $z_{0}$ near the equator), there is a limited vertical distance over which the waves can propagate before inequality (14) is violated. To focus thoughts, let us again consider upward propagating wave packets. Since $\Omega \ell /\left(B^{*} / \sqrt{\rho \mu}\right)$ is large, say 30 or greater, this critical height $z_{c}$ corresponds to

$$
\frac{B_{0}\left(z_{c}\right)}{\left|B_{0}\left(z_{0}\right)\right|}=\sqrt{1+\frac{2 \Omega}{\varpi_{B}\left(z_{0}\right)}} \approx \sqrt{\frac{2 \Omega}{\varpi_{B}\left(z_{0}\right)}} .
$$

We might term this critical height the wave ceiling, and for a linear variation in $B_{0}(z)$ it evidently scales as

$$
\frac{z_{c}}{\left|z_{0}\right|} \sim \frac{\Omega \ell}{B_{0}\left(z_{c}\right) / \sqrt{\rho \mu}} \text {. }
$$

Clearly, for $z_{0} \ll L$, the wave packets will traverse only a modest distance before being arrested, with the wave energy trapped in the 

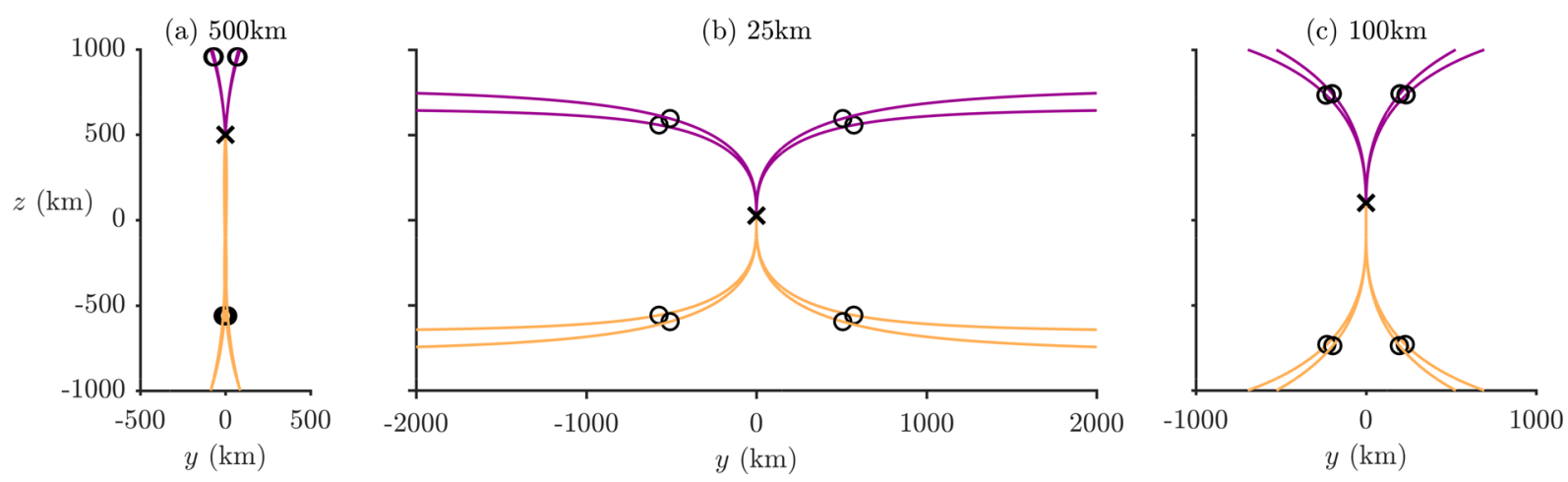

Figure 3. Rays dispersing from a small, localized buoyant anomaly of scale $\ell$. The trajectories shown are for the case of $B_{0}(z)=2 B^{*} z / L, \ell \approx \pi /\left|\mathbf{k}_{\perp}\right|=10 \mathrm{~km}$, $L=2000 \mathrm{~km}$, a normalized field strength of $\left(B^{*} / \sqrt{\rho \mu}\right) / \Omega \ell=0.033$ and a rotation rate equal to that of the Earth. The launch positions, denoted by $\mathbf{x}$, are (a) $z_{0}=500 \mathrm{~km}$, (b) $z_{0}=25 \mathrm{~km}$ and (c) $z_{0}=100 \mathrm{~km}$; northward (southward) trajectories are coloured purple (orange). The circles indicate the passage of (a) 0.1 , (b) $10 \mathrm{yr}$ and (c) $1 \mathrm{yr}$.
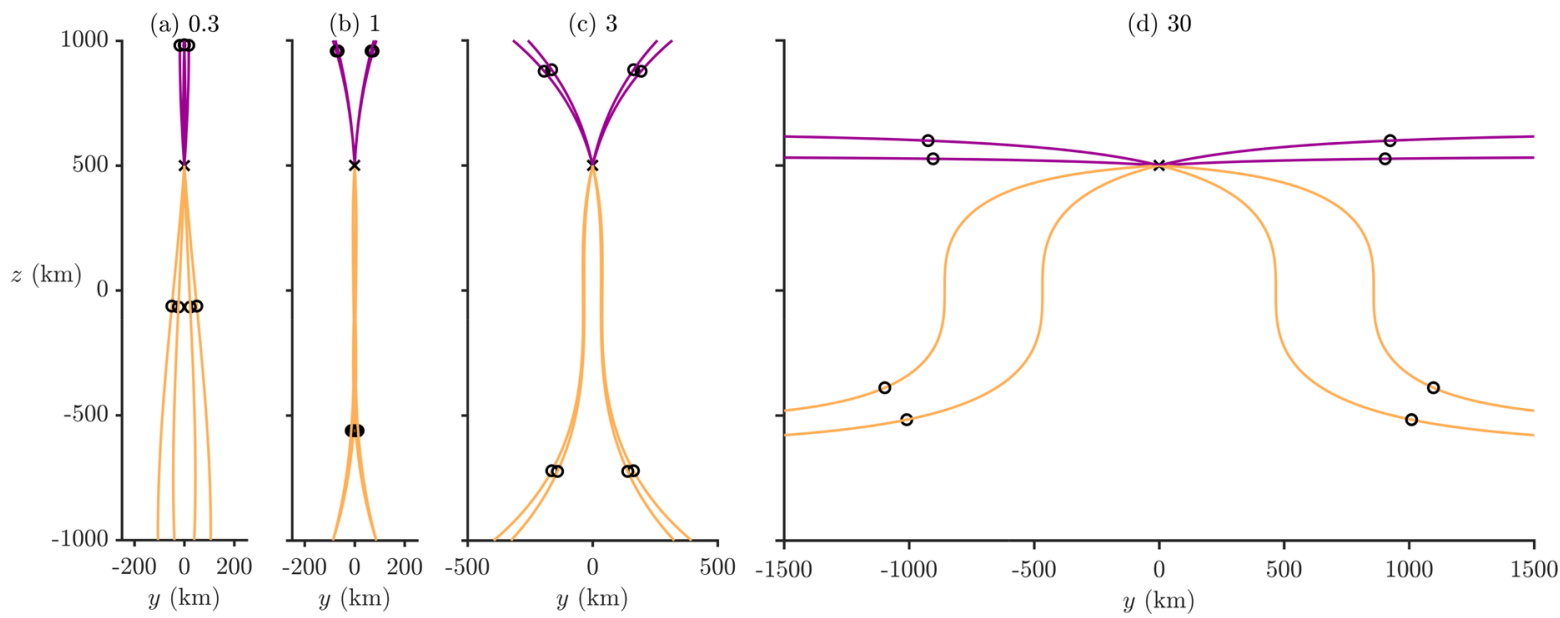

Figure 4. Ray dispersing from a small, localized source. The parameters are the same as for Fig. 3(a), except that, at launch, (a) $\varpi_{B} / \varpi_{0}=0.3$ (weakly modified inertial waves), (b) $\varpi_{B} / \varpi_{0}=1$ (inertial-Alfvén waves), (c) $\varpi_{B} / \varpi_{0}=3$ and (d) $\varpi_{B} / \varpi_{0}=30$ (magnetostrophic waves). The launch position, denoted by $\mathbf{x}$, is $z_{0}=500 \mathrm{~km}$ and the circles indicate the passage of (a) 0.04 , (b) 0.1 , (c) 0.5 and (d) $30 \mathrm{yr}$. Colours as Fig. 3.

equatorial regions. As noted by Acheson (1972), the wave ceiling corresponds to a critical layer at which $k_{\|} \gg k_{\perp}, k_{\|} / k \rightarrow 1$ and the axial component of the group velocity in (6) goes to zero. This is illustrated in Fig. 3(b) for a launch position of $z=25 \mathrm{~km}$, all other parameters being unchanged. The two sets of trajectories shown in the figure correspond to: (i) $\mathbf{k}_{\perp}$ aligned with $\mathbf{B}_{0}$, and (ii) $\mathbf{k}_{\perp}$ and $\mathbf{B}_{0}$ misaligned by a factor of $\pi / 4$. In both cases the existence of a wave ceiling is evident. (A misalignment of $\mathbf{k}_{\perp}$ and $\mathbf{B}_{0}$ is equivalent to reducing the effective value of $\mathbf{B}_{0}$; this does not alter $\mathbf{c}_{g \Omega}$ in (6), but does reduce the horizontal Alfvén velocity and $\varpi_{0}$.) Note that the circles on the rays in Fig. 3(b) indicate the passage of $10 \mathrm{yr}$, as opposed to $0.1 \mathrm{yr}$ in Fig. 3(a). The time scale is much longer because the lateral propagation of wave energy is associated with slow magnetostrophic waves.

In the interest of completeness, an intermediate case of $z_{0}=100 \mathrm{~km}$ (with all other parameters unchanged), is shown in Fig. 3(c). This time there is no wave ceiling below $1000 \mathrm{~km}$, although the wave packets acquire a significant horizontal component of group velocity. The circles now mark the passage of $1 \mathrm{yr}$.

Finally, it is of interest to consider the fate of wave packets which do not belong to the primary rays emitted from the source, that is, not launched with frequency $\varpi_{0}=\varpi_{B}$. Fig. 4(a) shows waves launched as weakly modified inertial waves in which $\varpi_{B} / \varpi_{0}=0.30$ and $\varpi_{0}=1.10 \varpi_{\Omega}$, Fig. 4(c) shows waves which are almost, but not quite, magnetostrophic at launch, corresponding to $\varpi_{B} / \varpi_{0}=3$ and $\varpi_{0} \approx 0.89 \varpi_{B}^{2} / \varpi_{\Omega}$, and Fig. $4(\mathrm{~d})$ shows waves which are strictly magnetostrophic, corresponding to $\varpi_{B} / \varpi_{0}=30$ and $\varpi_{0} \approx \varpi_{B}^{2} / \varpi_{\Omega}$. The circles in Figs 4(a)-(d) indicate the passage of $0.04,0.1,0.5$ and $30 \mathrm{yr}$, respectively. The relative sluggishness of magnetostrophic waves in (d) is evident, as is their tendency to spread horizontally.

\section{RAYS NEAR THE CROSSOVER POINT}

There is one technical issue associated with this model problem that needs to be clarified. While the primary rays are generated at the crossover point in Fig. $2\left(k_{\|}=0, \varpi_{0}=\varpi_{B}\right)$, wave packets launched away from this point may be required to pass through it as they propagate. However, the usual assumption of a gradually varying background, which underpins all of ray tracing theory, ceases to hold at this particular location, since we cannot satisfy the requirement that $k_{\|} L \gg 1$. Nevertheless, as with waves near a caustic, the difficulty can be remedied by a local analysis which smooths over the difficulties at the crossover point $k_{\|}=0$. This then allows conventional ray tracing methods to be applied either side of the crossover point (Lighthill 1978). 
We proceed as follows. Since we have homogeneity in transverse planes we can look for solution of (3) of the form $\mathbf{u}=$ $\hat{\mathbf{u}}(z) \exp \left\{i\left(\mathbf{k}_{\perp} \cdot \mathbf{x}_{\perp}-\varpi t\right)\right\}$, which yields

$\frac{\mathrm{d}^{2} \hat{\mathbf{u}}}{\mathrm{d} z^{2}}+\frac{\left(\varpi^{2}-\varpi_{B}^{2}\right)^{2} k_{\perp}^{2}}{4 \Omega^{2} \varpi^{2}-\left(\varpi^{2}-\varpi_{B}^{2}\right)^{2}} \hat{\mathbf{u}}=0$.

In the vicinity of the crossover point (or turning point) $\varpi=\varpi_{B}\left(z_{\times}\right)$, this simplifies to

$$
\frac{\mathrm{d}^{2} \hat{u}}{\mathrm{~d} \hat{z}^{2}}+\frac{4 \hat{z}^{2}}{\hat{L}^{4}} \hat{u}=0, \quad \frac{1}{\hat{L}^{2}}=\frac{k_{\perp}}{2 \Omega}\left|\frac{\mathrm{d} \varpi_{B}}{\mathrm{~d} z}\right|_{z_{\times}},
$$

where $\hat{z}=z-z_{\times}$is the distance from the turning point and $\hat{u}$ is any one component of $\hat{\mathbf{u}}$. The general solution of (18) is

$\hat{u}^{ \pm}=\sqrt{\frac{|\hat{z}|}{\hat{L}}}\left[A^{ \pm} J_{\frac{1}{4}}\left(\frac{\hat{z}^{2}}{\hat{L}^{2}}\right)+C^{ \pm} J_{-\frac{1}{4}}\left(\frac{\hat{z}^{2}}{\hat{L}^{2}}\right)\right]$,

where $J_{\frac{1}{4}}$ and $J_{-\frac{1}{4}}$ are the usual Bessel functions and \pm indicates solutions above and below the turning point; the coefficients $A^{+}$and $C^{+}$apply above it and $A^{-}$and $C^{-}$below.

We now determine the coefficients as follows. Expanding the Bessel functions for small argument yields, after a little algebra,

$\hat{u}^{ \pm}\left(\frac{\hat{z}}{\hat{L}} \rightarrow 0\right)=\frac{2^{1 / 4}}{\Gamma(3 / 4)} C^{ \pm}+\frac{|\hat{z}| / \hat{L}}{2^{1 / 4} \Gamma(5 / 4)} A^{ \pm}+O\left(|\hat{z} / \hat{L}|^{4}\right)$.

Continuity across $\hat{z}=0$ now requires that $C^{+}=C^{-}=C$ and $A^{+}=-A^{-}$, which is sufficient to ensure that $\hat{u}$ and all its derivatives are continuous. Moreover, combining (1) with (2) in the form

$$
\left[\frac{\partial^{2}}{\partial t^{2}}-\frac{1}{\rho \mu}\left(\mathbf{B}_{0} \cdot \nabla\right)^{2}\right] \nabla \times \mathbf{u}=(2 \boldsymbol{\Omega} \cdot \nabla) \frac{\partial \mathbf{u}}{\partial t}
$$

demands that when $\varpi=\varpi_{B}\left(z_{\times}\right), \mathrm{d} \hat{u} / \mathrm{d} \hat{z}=0$ at $\hat{z}=0$. This implies $A^{ \pm}=0$, and the solution is therefore symmetric about $\hat{z}=0$, taking the form

$$
\hat{u}=C \sqrt{\frac{|\hat{z}|}{\hat{L}}} J_{-\frac{1}{4}}\left(\frac{\hat{z}^{2}}{\hat{L}^{2}}\right) .
$$

Finally, replacing the Bessel function by its asymptotic approximation at large argument shows that, for large $\hat{z}^{2} / \hat{L}^{2}$,

$\hat{u} \approx C \sqrt{\frac{2 \hat{L}}{\pi|\hat{z}|}} \cos \left(\frac{\hat{z}^{2}}{\hat{L}^{2}}-\frac{\pi}{8}\right)$,

which is the limiting form of the Wentzel-Kramers-Brillouin (WKB) solution to the problem in hand (Hinch 1991). A comparison of the exact and WKB solutions is shown in Fig. 5. Note that the singularity in the WKB approximation at $\hat{z} \rightarrow 0$, indicative of the violation of $k_{\|} L \gg 1$, is smoothed over in the exact solution within a distance of order $\hat{L}$. Moreover, the $|\hat{z}|^{-1}$ decay of $\hat{u}^{2}$ exhibited by the WKB solution is a direct manifestation of the defocusing of the radiation as it moves away from the turning point. Finally, we observe that there is no reflection of energy at the turning point, as would be expected since the axial group velocity remains finite at $\hat{z}=0$.

\section{IMPLICATIONS FOR WAVE DISPERSION IN THE CORE OF THE EARTH}

In the core of the Earth the buoyancy flux outside the tangent cylinder (an imaginary cylinder that circumscribes the solid inner core)

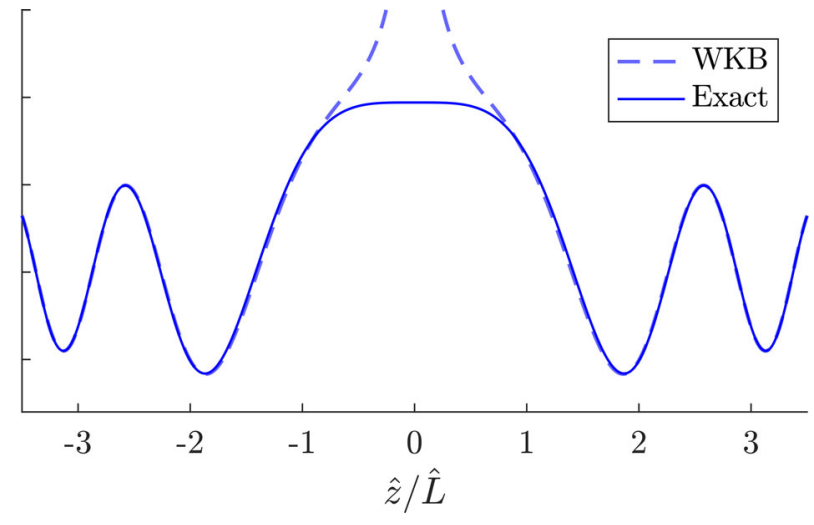

Figure 5. Comparison of the exact and WKB solutions (22) and (23).

tends to be concentrated in and around the equatorial regions (see, e.g. Sakuraba \& Roberts 2009) and it is argued in Davidson \& Ranjan (2015) that the waves emanating from such regions may play an important role in magnetic field generation. The buoyant anomalies that trigger such waves probably exhibit a wide range of scales (Roberts \& King 2013), perhaps all the way down to a few kilometres (Davidson 2016).

The model problem discussed above is somewhat idealized, intended simply to explore the influence of a spatially varying magnetic field on the dispersive properties of inertial-Alfvén waves. In this section we consider the implications of this behaviour for the core of the Earth. Let us start by considering a more realistic distribution of $B_{0}(z)$. Since the azimuthally averaged east-west field in the core is antisymmetric about $z=0$, and zero at both the equator and core-mantle boundary, perhaps a slightly more plausible distribution is

$B_{0}(z)=B^{*} \sin \left(\frac{\pi z}{L}\right), \quad-L<z<L$,

where $L$ is indicative of the core radius.

Keeping all other parameters the same as in Fig. 3, the corresponding ray paths are shown in Fig. 6 for the launch positions of (a) $z_{0}=500 \mathrm{~km}$, (b) $z_{0}=25 \mathrm{~km}$ and (c) $z_{0}=100 \mathrm{~km}$. As before, the circles on the ray trajectories indicate the passage of $0.1 \mathrm{yr}$ in (a), $10 \mathrm{yr}$ in (b) and $1 \mathrm{yr}$ in (c). Although the details differ from those of Fig. 3, the general trend remains the same, with wave packets launched near the equator confined by a wave ceiling, whilst those launched far from the equator maintain a predominantly axial trajectory. The primary difference between the two figures lies in the intermediate case of $z_{0}=100 \mathrm{~km}$; for a linear distribution of $B_{0}(z)$ the rays simply diverge as the magnetic field increases, whereas for the sinusoidal distribution they eventually revert to something close to a vertical group velocity as the magnitude of $B_{0}(z)$ falls back towards $B_{0}\left(z_{0}\right)$. (There is also some evidence of this behaviour in the $z_{0}=500 \mathrm{~km}$ case.)

It is evident from (16) that waves launched below

$\left|z_{0}\right| \sim \frac{B^{*} / \sqrt{\rho \mu}}{\Omega \ell} \frac{L}{2}$

are likely to be trapped in the equatorial regions and this corresponds to a $z_{0}$ of around $30-40 \mathrm{~km}$ for the parameters used in Fig. 6, that is, close to the equator. This is consistent with the trajectories shown in Fig. 6(b). Note that those waves which are trapped near the equator develop a large vertical wavenumber, $\left|k_{\|}\right| \gg\left|k_{\perp}\right|$, and so will be subject to particularly intense Ohmic dissipation. In short, such waves are not only trapped at low latitudes, but likely to be rapidly eradicated. 

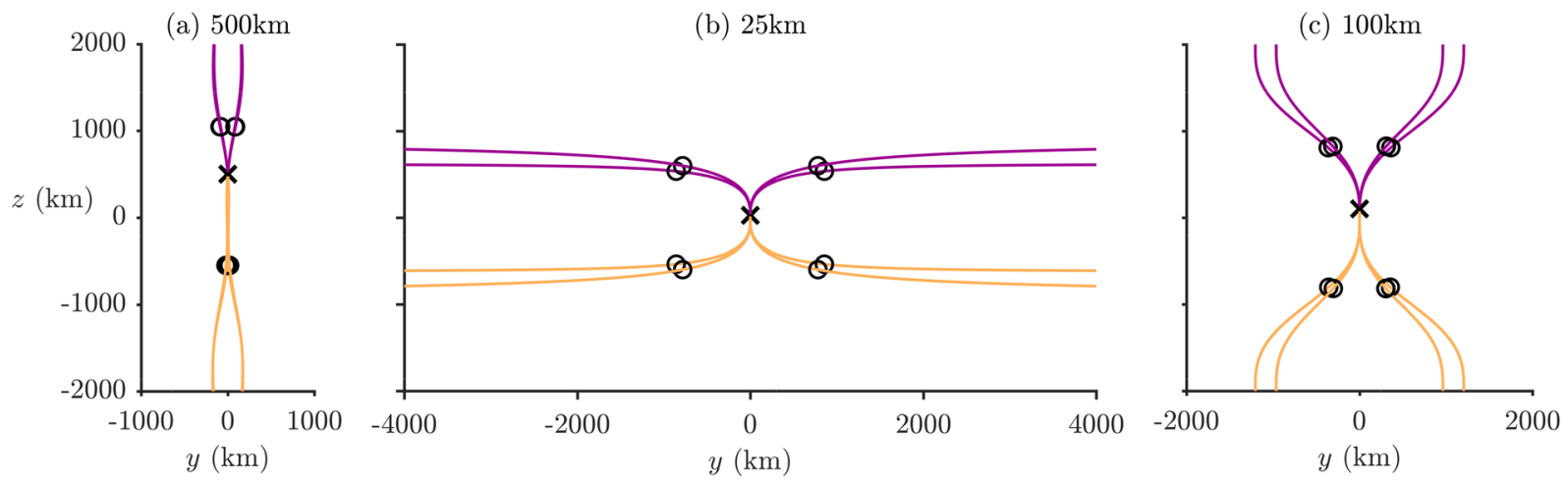

Figure 6. Rays dispersing from a localized source when the background field $B_{0}(z)$ varies as a sinusoid according to (24). The colours and all parameters apart from $B_{0}(z)$ are the same as for Fig. 3.

Conversely, the large value of $\Omega \ell /\left(B^{*} / \sqrt{\rho \mu}\right)$ in the core of the Earth, combined with (11), ensures that $\left|k_{\|}\right| / k \ll 1$ whenever waves are launched an appreciable distance from the equator, that is, $\left|z_{0}\right| / L=O(1)$. The dispersion in such cases is mostly along the rotation axis, consistent with the trajectories shown in Fig. 6(a). So we conclude that, for most launch locations, the trajectories of the wave packets remain more or less aligned with the rotation axis, with the axial group speed being of the order of the fast inertial wave speed, $c_{g z} \sim \Omega / k_{\perp}$. As discussed in Section 2.2, such waves satisfy $\left|\varpi_{\Omega}\right| \sim\left|\varpi_{B}\right|$ and so cannot formally be classified as hybrid inertial-Alfvén waves $\left(\left|\varpi_{\Omega}\right| \ll\left|\varpi_{B}\right|\right)$, magnetostrophic waves $\left(\left|\varpi_{\Omega}\right| \gg\left|\varpi_{B}\right|\right)$, or even weakly modified inertial waves $\left(\left|\varpi_{\Omega}\right| \gg\left|\varpi_{B}\right|\right)$. We shall refer to them instead as intermediate magneto-Coriolis $(M C)$ waves, a generalization of the nomenclature fast/slow MC waves commonly used to describe inertial/magnetostrophic waves (Finlay et al. 2010).

The general picture that emerges, then, is the following. At least four sets of waves contribute to the axial dispersion of wave energy from a localized source: weakly modified inertial waves, magnetostrophic waves, inertial-Alfvén waves and intermediate MC waves. The first set is characterized by $c_{g z} \approx 2 \Omega / k_{\perp}$ and the ranking $\left|\varpi_{\Omega}\right| \gg\left|\varpi_{B}\right|$, the second by $c_{g z} \ll \Omega / k_{\perp}$ and $\left|\varpi_{\Omega}\right| \gg\left|\varpi_{B}\right|$, the third by $c_{g z} \approx \Omega / k_{\perp},\left|\varpi_{\Omega}\right| \ll\left|\varpi_{B}\right|$ and $\boldsymbol{\Omega} \cdot \boldsymbol{k} \approx 0$, and the fourth by $c_{g z} \sim \Omega / k_{\perp}$ and $\left|\varpi_{\Omega}\right| \sim\left|\varpi_{B}\right|$. Because the Alfvén velocity is relatively slow, in the sense that $\Omega \ell \gtrsim 30|\mathbf{B}| / \sqrt{\rho \mu}$, the intermediate MC waves propagate mainly along the rotation axis, with $\left|k_{\|}\right| / k \ll$ 1 and $\mathbf{c}_{g \Omega}$ almost aligned with $\boldsymbol{\Omega}$, though they do retain some group velocity component along magnetic field lines. These four classes of waves - weakly modified inertial waves, magnetostrophic waves, inertial-Alfvén waves and intermediate MC waves-are shown in Fig. 7.

Close to the source the dispersion pattern is like that shown in Fig. 1(a), with much of the energy carried by axially propagating inertial-Alfvén waves, which are self-focussing and have an axial group velocity of $c_{g z} \approx \Omega / k_{\perp}$. However, a significant fraction of the energy is also carried by slightly off-axis inertial waves, which have the faster group speed of $c_{g z} \approx 2 \Omega / k_{\perp}$ (Davidson et al. 2006, BD16). Over a longer time scale the Alfvén component of the inertialAlfvén waves near the source transports energy horizontally.

Far from the source, on the other hand, the inertial-Alfvén waves typically convert into intermediate $\mathrm{MC}$ waves and acquire an offaxis component of $\mathbf{c}_{g \Omega}$. (In principle, some of the wave packets may be trapped by a wave ceiling, but in practice this would occur only when the source is particularly small and located close to the equator.) Intermediate MC waves satisfy $\left|\varpi_{\Omega}\right| \sim\left|\varpi_{B}\right|$ and disperse energy along the rotation axis in a manner reminiscent of

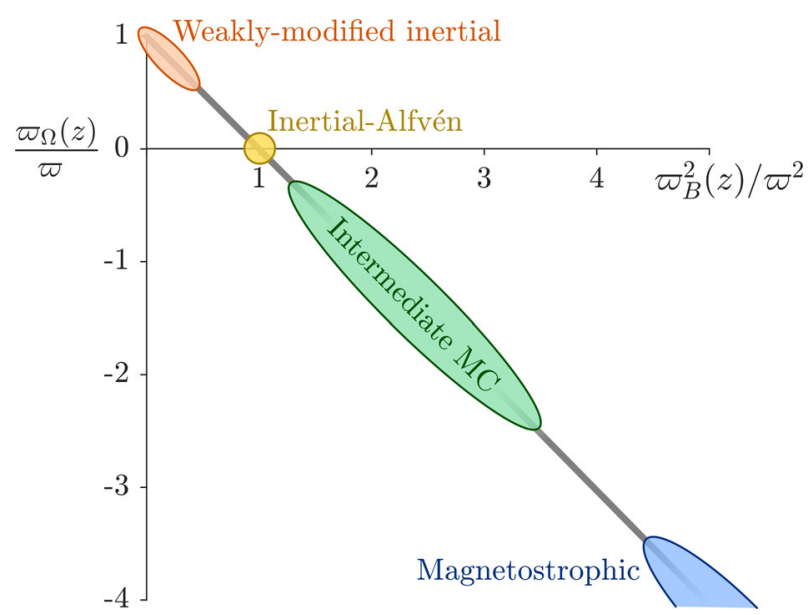

Figure 7. The relative frequencies of weakly modified inertial waves, magnetostrophic waves, inertial-Alfvén waves and intermediate MC waves.

low-frequency inertial waves. However, they also initiate horizontal Alfvén waves and transport magnetic helicity. We conclude, therefore, that quasi-geostrophy in the core is likely to be maintained through a combination of slightly off-axis inertial waves, which satisfy $\left|\varpi_{\Omega}\right| \gg\left|\varpi_{B}\right|$, and intermediate MC waves which satisfy $\left|\varpi_{\Omega}\right| \sim\left|\varpi_{B}\right|$ and have an axial group velocity comparable to that of inertial waves.

Finally, in order to convey the complexity of the dispersion pattern that can develop, in Fig. 8 we have superimposed rays dispersing from three localized sources at slightly different longitudes and with the background field varying as (24). As before, we take $\ell=10 \mathrm{~km}, L=2000 \mathrm{~km}$, a normalized field strength of $\left(B^{*} / \sqrt{\rho \mu}\right) / \Omega \ell=0.033$, and a rotation rate equal to that of the Earth. The waves are launched at latitudes of $z_{0}=25,100$, $500 \mathrm{~km}$ and for each source we allow for launch frequencies of $\varpi_{B} / \varpi_{0}=0.3$ (weakly modified inertial waves), $\varpi_{B} / \varpi_{0}=1$ (inertial-Alfvén waves) and $\varpi_{B} / \varpi_{0}=3$. Broadly speaking the waves fall into one of two categories; some rays remain trapped in the equatorial regions, say $\left|z_{0}\right|<100 \mathrm{~km}$, but those which escape the region propagate towards the mantle as intermediate MC waves $\left(\left|\varpi_{\Omega}\right| \sim\left|\varpi_{B}\right|\right)$, or else weakly modified inertial waves $\left(\left|\varpi_{\Omega}\right| \gg\left|\varpi_{B}\right|\right)$. As noted earlier, those waves that remain trapped near the equator are likely to be subject to strong Ohmic dissipation.

Explicit discussion of the effect of a finite magnetic diffusivity is beyond the scope of this work, as is the treatment of other largescale magnetic field components. While the axial field is likely subordinate to the rotation, the spatially varying radial field will 


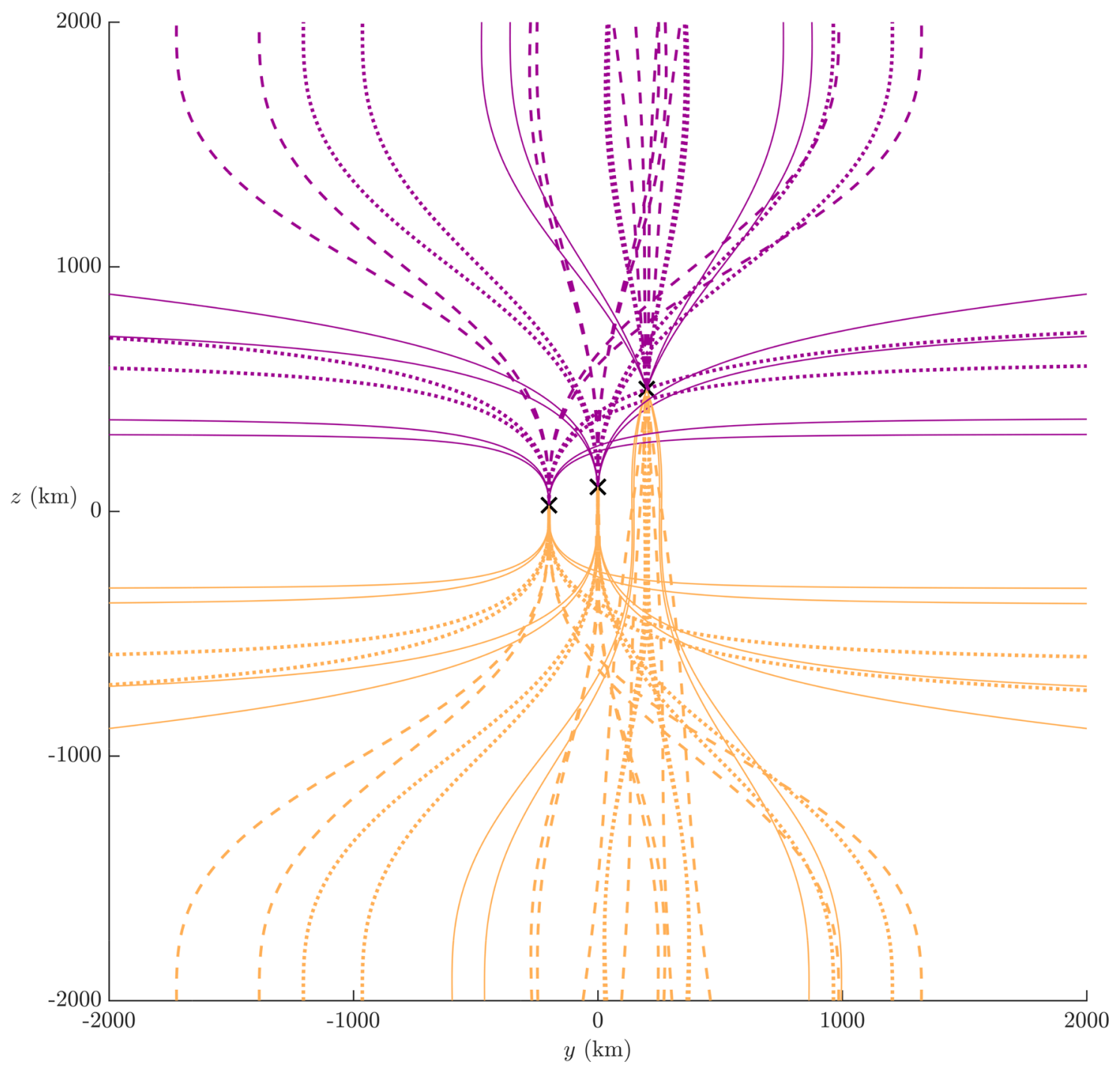

Figure 8. Rays dispersing from three localized sources with a sinusoidal background field. As in earlier figures, we take $\ell=10 \mathrm{~km}, L=2000 \mathrm{~km}$, a normalized field strength of $\left(B^{*} / \sqrt{\rho \mu}\right) / \Omega \ell=0.033$, and a rotation rate equal to that of the Earth. The launch positions, denoted by $\mathbf{x}$, are $z_{0}=25,100,500 \mathrm{~km}$ and at each source we allow for launch frequencies of $\varpi_{B} / \varpi_{0}=0.3$ (weakly modified inertial waves, dashed), $\varpi_{B} / \varpi_{0}=1$ (inertial-Alfvén waves, dotted) and $\varpi_{B} / \varpi_{0}=3$ (intermediate MC waves, solid). Colours as Fig. 3.

have some bearing on the ray paths; it allows for a transverse field which varies in direction as well as magnitude. (This is the situation considered by Acheson (1972) when studying the wave ceiling.) We defer discussion of this and other interesting cases to future investigations.

\section{CONCLUSIONS}

There exists a zoo of wave types that can transport helicity across the Earth's core, including weakly modified inertial waves, magnetostrophic waves, inertial-Alfvén waves, and intermediate MC waves, as shown in Fig. 7. Weakly modified inertial waves are characterized by $c_{g z} \approx 2 \Omega / k_{\perp}$ and $\left|\varpi_{\Omega}\right| \gg\left|\varpi_{B}\right|$, magnetostrophic waves by $c_{g z} \ll \Omega / k_{\perp}$ and $\left|\varpi_{\Omega}\right| \gg\left|\varpi_{B}\right|$, inertial-Alfvén waves by $c_{g z} \approx \Omega / k_{\perp},\left|\varpi_{\Omega}\right| \ll\left|\varpi_{B}\right|$ and $\boldsymbol{\Omega} \cdot \boldsymbol{k} \approx 0$, and intermediate MC waves by $c_{g z} \sim \Omega / k_{\perp}$ and $\left|\varpi_{\Omega}\right| \sim\left|\varpi_{B}\right|$. Inertial-Alfvén waves cannot be sustained for long when the ambient magnetic field is non-uniform, and magnetostrophic waves preferentially send energy and helicity along the magnetic field lines, rather than along the rotation axis. On the other hand, because $\Omega \ell \gtrsim 30|\mathbf{B}| / \sqrt{\rho \mu}$ in the Earth's core, the intermediate MC waves propagate more or less along the rotation axis, with $\left|k_{\|}\right| / k \ll 1$ and $\mathbf{c}_{g \Omega}$ almost aligned with $\boldsymbol{\Omega}$, as do low-frequency inertial waves. We conclude, therefore, that the maintenance of a quasi-geostrophic flow in the presence of continual stirring by buoyancy forces is most likely to be associated with weakly modified inertial waves and intermediate MC waves. Both of these wave types are also good candidates for the maintenance of an asymmetric helicity distribution about the equator, with northward propagating waves carrying negative helicity and south travelling waves positive helicity; such an asymmetric helicity distribution is beneficial to the maintenance of a dynamo (Davidson 2014).

\section{ACKNOWLEDGEMENTS}

This work was undertaken with funding from an EPSRC doctoral studentship. The authors thank Avishek Ranjan and two anonymous reviewers for their helpful comments. 


\section{REFERENCES}

Acheson, D.J., 1972. The critical level for hydromagnetic waves in a rotating fluid, J. Fluid Mech., 53, 401-415.

Bardsley, O.P. \& Davidson, P.A., 2016. Inertial-Alfvén waves as columnar helices in planetary cores, J. Fluid Mech., 805, doi:10.1017/jfm.2016.577. (BD16)

Davidson, P.A., 2014. The dynamics and scaling laws of planetary dynamos driven by inertial waves, Geophys. J. Int., 198(3), 1832-1847.

Davidson, P.A., 2016. Dynamos driven by helical waves: scaling laws for numerical dynamos and for the planets, Geophys. J. Int., 207(2), 680-690.

Davidson, P.A. \& Ranjan, A., 2015. Planetary dynamos driven by helical waves: Part 2, Geophys. J. Int., 202, 1646-1662.

Davidson, P.A., Staplehurt, P.J. \& Dalziel, S.B., 2006. On the evolution of eddies in a rapidly-rotating system, J. Fluid. Mech., 557, 135-144.

Finlay, C.C., Dumberry, M., Chulliat, A. \& Pais, M.A., 2010. Short timescale core dynamics: theory and observations, Space Sci. Rev., 155, $177-218$.
Gillet, N., Jault, D., Canet, E. \& Fournier, A., 2010. Fast torsional waves and strong magnetic field within the Earth's core, Nature, 465, 74-77.

Hinch, E.J., 1991. Perturbation Methods, Cambridge Univ. Press.

Jault, D., 2008. Axial invariance of rapidly varying diffusionless motions in the Earth's core interior, Phys. Earth planet Inter., 166, 67-76.

Lighthill, M.J., 1978. Waves in Fluids, Cambridge Univ. Press.

Moffatt, H.K., 1970. Dynamo action associated with random inertial waves in a rotating conducting fluid, J. Fluid Mech., 44(4), 705-719.

Moffatt, H.K., 1978. Magnetic Field Generation in Electrically Conducting Fluids, Cambridge Univ. Press.

Olson, P., 1981. A simple physical model of the terrestrial dynamo, J. geophys. Res., 86, 10 875-10 882.

Roberts, P.H. \& King, E.M., 2013. On the genesis of the Earth's magnetism, Rep. Prog. Phys., 76(9), doi:10.1088/0034-4885/76/9/096801.

Sakuraba, A. \& Roberts, P.H., 2009. Generation of a strong magnetic field using uniform heat flux at the surface of the core, Nat. Geosci., 2, 802805 . 